\title{
Resolved Stellar Population Modeling
}

\author{
Antonio Aparicio ${ }^{1,2}$, Sebastián L. Hidalgo ${ }^{3}$, Carme Gallart ${ }^{2}$ \\ and Santi Cassisi ${ }^{4}$ \\ ${ }^{1}$ Departamento de Astrofísica, Universidad de La Laguna, Tenerife, Canary Islands, Spain \\ email: antapaj@iac.es \\ ${ }^{2}$ Instituto de Astrofísica de Canarias, Vía Láctea, La Laguna, Tenerife, Canary Islands, Spain \\ ${ }^{3}$ University of Minnesota, Minneapolis, MN, USA \\ ${ }^{4}$ INAF - Osservatorio di Collurania, Teramo, Italy
}

\begin{abstract}
IAC-pop is a code designed to solve the star formation history (SFH) of a complex stellar population system, like a galaxy, from the analysis of the color-magnitude diagram (CMD). It uses a genetic algorithm to minimize a $\chi^{2}$ merit function comparing the stellar distribution of an observed CMD and the stellar distributions of CMDs arising from linear combination of simple synthetic populations. To this purpose, IAC-star is used to compute a single seminal synthetic CMD from which the simple populations and their corresponding CMD stellar distributions are extracted.

In the current version, IAC-pop provides two different estimates of the errors in the SFH. It is offered for free use and can be downloaded from the site www.iac.es/iac-pop.html.
\end{abstract}

Keywords. galaxies: dwarf, galaxies: irregular, (galaxies:) Local Group, galaxies: stellar content, (stars:) Hertzsprung-Russell diagram, stars: statistics, methods: numerical

\section{Introduction}

The color-magnitude diagram (CMD) is the best tool to study and derive the star formation history of a galaxy. Deep enough CMDs display stars born all over the lifetime of the galaxy and are indeed fossil records of the Star Formation History (SFH). But an accurate SFH can only be obtained through sophisticated techniques of CMD synthesis. In Aparicio \& Gallart (2004) we presented IAC-star, a code for synthetic CMD computation. In short, the algorithm is intended to be as general as possible and allows a variety of inputs for the initial mass function (IMF), star formation rate, metallicity law and binariety. Stars with age and metallicity following a continuous distribution are computed through interpolation in a stellar evolution library, providing synthetic CMDs with smooth, realistic stellar distributions. Several stellar evolution and bolometric correction libraries can be used.

In this contribution we present IAC-pop, an algorithm based on a genetic code and designed for deriving the SFR and the Chemical Enrichment Law (CEL) of a system from the comparison of its CMD stellar distribution with the stellar distribution in a single template synthetic CMD.

\section{Basic definitions}

The SFH is composed by several pieces of information. For simplicity we will adopt here the following approach: considering that time and metallicity are the most important variables in the problem, we define the $\mathrm{SFH}$ as a function $\psi(t, z)$ such that $\psi(t, z) \mathrm{d} t \mathrm{~d} z$ is the number of stars formed at time $t^{\prime}$ in the interval $t<t^{\prime} \leqslant t+\mathrm{d} t$ and with metallicity $z^{\prime}$ in the interval $z<z^{\prime} \leqslant z+\mathrm{d} z$, per unit of time and metallicity. There are several other 


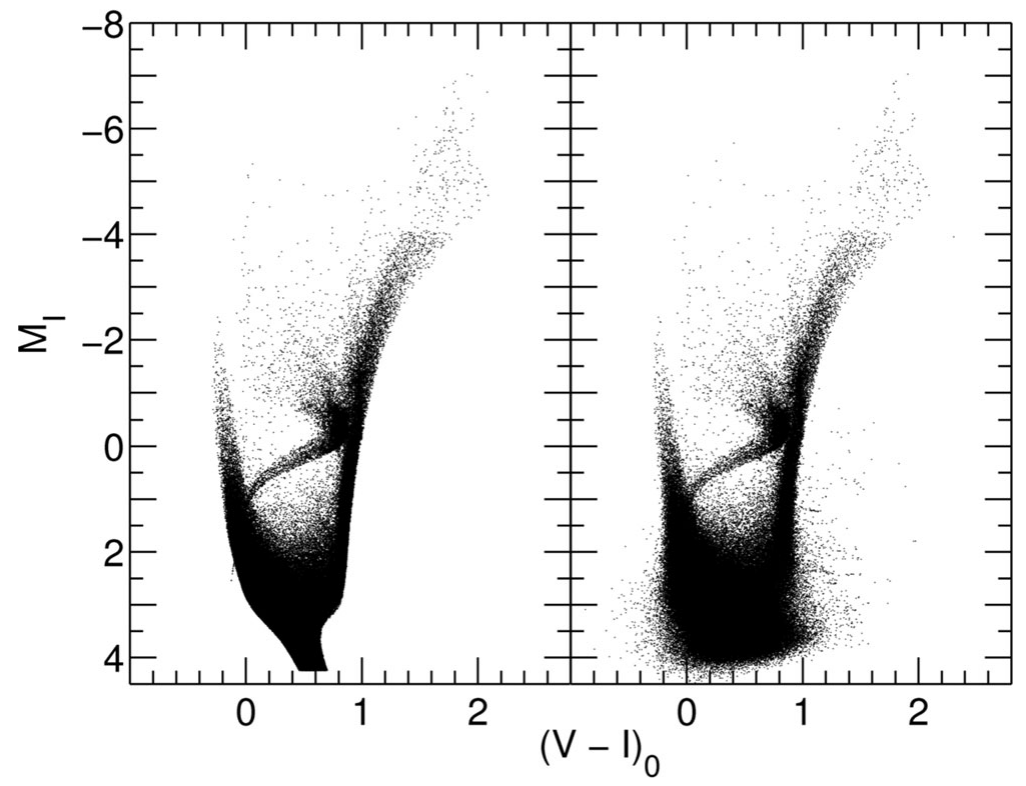

Figure 1. Distortion introduced in a synthetic CMD by observational effects. Left panel shows the synthetic CMD before introducing the observational effects. Right panel shows the same after simulations of observational effects according to Aparicio \& Gallart (1995).

functions and parameters related to the SFH, that we will consider here as auxiliary. The Initial Mass Function (IMF), $\phi(m)$ and a function accounting for the frequency and relative mass distribution of binary stars, $\beta(f, q)$ are the main ones. The solution found for the SFH depends on the assumptions made for $\phi(m)$ and $\beta(f, q)$. Other parameters affecting the solution of $\psi(t, z)$ are the distance and reddening, including differential reddening.

The strongest limitation in the information that can be retrieved by the empirical data, is produced by the observational effects. These include all the factors affecting and distorting the CMD, namely the signal-to-noise limitations, the defects of the detector and the crowding and blending between stars. The consequences are loss of stars, changes in measured stellar colors and magnitudes, and external errors larger and more difficult to control than internal ones. Figure 1 shows the distortion introduced in a synthetic CMD by observational effects, simulated according to Aparicio \& Gallart (1995). It must be kept in mind that IAC-pop does not account for the observational effects that must be considered and simulated in advance by the user into the synthetic models.

In the following, we will concentrate in the determination of $\psi(t, z)$ on the understanding that all the remaining functions and parameters are externally checked, and that proper assumptions are made for them.

\section{Parameterizing the CMD}

The procedure used by IAC-pop is based on the approach introduced separately by Aparicio et al. (1997) and Dolphin (1997). An important improvement is that IAC-pop makes use of a genetic algorithm (pikaia; Charbonneau 1995) for an efficient searching of SFH solutions. The method is based on the following steps:

(a) The synthetic CMD computation code IAC-star is used to generate a single mother synthetic CMD with a large number of synthetic stars with ages and metallicities 
uniformly distributed over the full interval of variation of $\psi(t, z)$ in time and metallicity. This represents a constant SFR as a function of time with equally probable metallicity, within a given range, for each age. Observational effects (crowding, blending, external errors, etc) should be simulated in the synthetic CMD.

(b) The former synthetic stars are distributed in an array of partial or simple models (see Fig. 2). Each contains the stars within small intervals of age and metallicity. The set of partial models can be considered as a base of an $n \times m$-dimensional vector space, where $n$ and $m$ are respectively the number of age and metallicity intervals.

(c) A set of boxes is defined in the CMD (Fig. 3). In practice, two approaches may be used: an uniform grid and an a la carte grid. The uniform grid is more objective and less dependent on human criteria. The a la carte grid allows different sampling of well and poorly known stellar evolution phases.

(d) An array, $M_{i}^{j}$, containing the number of stars from partial model $i$ populating box $j$ is computed. The same operation is made in the observational CMD, producing a vector, $O^{j}$, containing the number of observed stars in box $j$. This step defines the parameterization of the CMD.

(e) Then the distribution of stars in the defined boxes can be calculated for any model $\mathrm{SFH}$ as a linear combination of the $M_{i}^{j}$ (it must be noted that $\alpha_{i} \geqslant 0$ ):

$$
M^{j}=A \sum_{i} \alpha_{i} M_{i}^{j}
$$

(f) The set of $\alpha_{i}$ parameters which results in a best matching of $M_{i}^{j}$ and the observational distribution $O^{j}$ can be found using a merit function, like $\chi^{2}$. In particular a reduced Mighell $\chi^{2}$ (Mighell 1999) is used, $\chi_{\nu}^{2}=\chi^{2} / \nu$, where $\nu=k-1$ is the number of freedom degrees, being $k$ the number of boxes used to parameterize the CMD. Minimization of $\chi_{\nu}^{2}$ provides the best solution as well as a test on whether it is also acceptable and a way to estimate errors for the solution. IAC-pop makes use of a genetic algorithm for the minimization of $\chi_{\nu}^{2}$. Considering the large number of dimensions of the problem $(n \times m)$, such an efficient solving procedure is required.

( $g$ ) The solution for the SFH can be written as:

$$
\psi(t, z)=A \sum_{i} \alpha_{i} \psi_{i}
$$

where $\psi_{i}$ refers to the partial model $i$, with $i$ taking values from 1 to $n \times m$, and $A$ is a scaling constant.

\section{Testing IAC-pop}

Self-consistency is a main requirement that methods to solve the SFH must fulfill. To test it, a synthetic CMD generated from some arbitrary SFH is solved blindly as if it was real data and the solution is compared with the input SFH. IAC-star (Aparicio \& Gallart 2004) was used to build the fake population CMD (fCMD hereafter). As input parameters, the stellar evolution library of Padua (summarized in Bertelli et al. 1994) and the Castelli \& Kurucz (2003) bolometric corrections were used. The number of stars in the fCMD were $10^{5}$ and the population was computed with constant SFR from 15 Gyr ago to present and a CEL according to the parameters $z_{0}=0.0001, z_{f}=0.008$, $\mu_{f}=0.2, \alpha=0, \lambda=1$, where $z_{0}$ (the initial metallicity), $z_{f}$ (the final metallicity), $\mu_{f}$ (the final gas mass fraction), $\alpha$ (in-fall parameter) and $\lambda$ (the outflow parameter) are usual parameters defining a CEL (see Aparicio \& Gallart 2004 for a complete description 


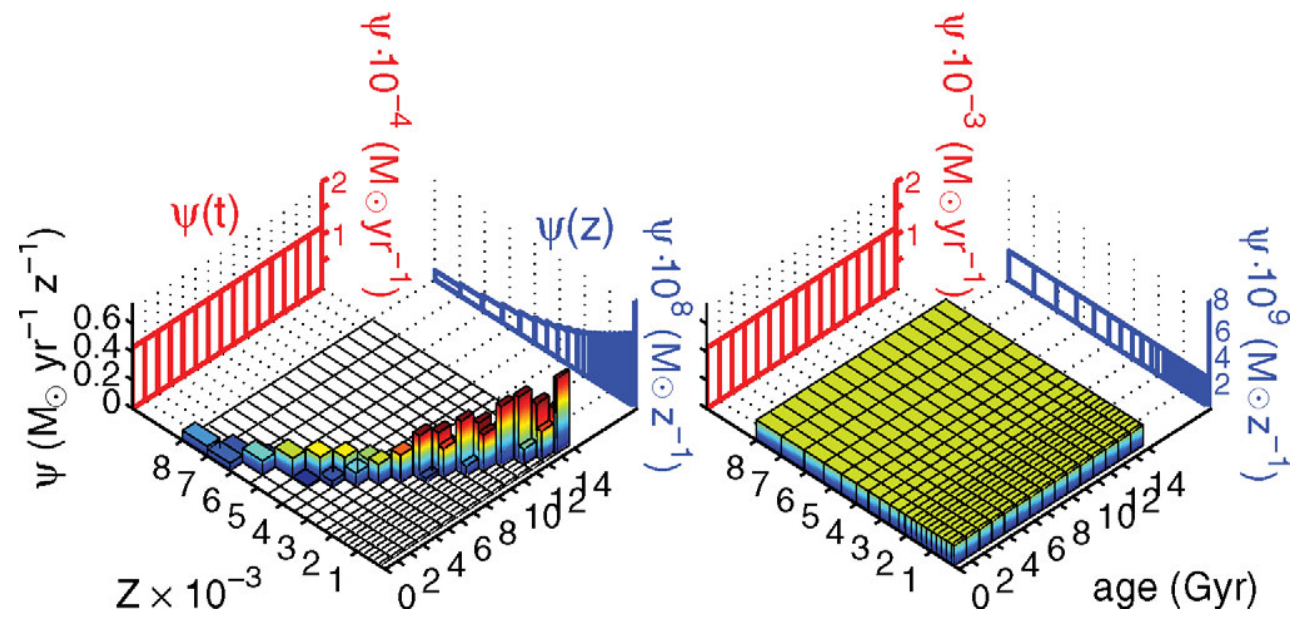

Figure 2. Left panel: the SFH $\psi(t, z)$ of the fake population. $\psi(t, z)$ is shown in the vertical axis while age and metallicity are shown in the horizontal ones. The volume of each bar over the age-metallicity plane gives the mass in stars that has been ever formed within the age-metallicity interval. Right panel: The SFH $\psi(t, z)$ of the mother seminal synthetic population. A constant $\psi(t, z)$ over the entire range of expected ages and metallicities is used. Both panels: the SFR as a function of time only $(\psi(t))$ and on metallicity only $(\psi(z))$ are shown (in red and blue, respectively in the electronic version of the paper). The $15 \times 20$ single stellar populations in which it has been divided for the SFH solving with IAC-pop are also shown.

of IAC-star). No binary stars were considered and the IMF by Kroupa, Tout \& Gilmore (1993) was used.

Figure 2, left panel, shows the SFH of the fake population. Age and metallicity have been divided into 15 and 20 intervals respectively. These intervals are the same defining the models used to solve for the SFH. The volume of each bar on the age-metallicity plane gives the mass that has been ever transformed into stars within the corresponding age-metallicity interval.

A mother synthetic CMD (sCMD hereafter) was calculated and used as the seminal information to solve the SFR of the fCMD. The input parameters given to IAC-star were the same as for the fake population except for the metallicity law which, in this case is an uniform distribution covering the full range of possible values for each age; i.e. stars of any age between 0 and 15 Gyr can have any metallicity between $z_{0}=0.0001$ and $z_{f}=0.008$. Function $\psi(t, z)$ for the seminal population is shown in figure 2 , right panel.

To parameterize the fCMD and sCMD, a set of boxes is used. We will only show the results for a box set uniformly distributed over the CMDs. Figure 3 shows the grid over-imposed on the fCMD and sCMD.

Figure 4 shows the $\psi(t, z)$ solution for two different tests. In the first one (Fig. 4, left panel), the stellar evolution library for the fake and the mother synthetic populations are the same. In the second one (Fig. 4, right panel), the fake population is computed using the Padua (Bertelli et al. 1994) library while the mother synthetic population is computed using the BaSTI-Teramo (Pietrinferni et al. 2004) population. The first case test the self-consistency of the method, i.e., the capability of IAC-pop to reproduce a given population. Departures between the fake population of Fig. 2, left panel, and the solution shown in Fig. 4, left panel, show the intrinsic limitation of IAC-pop. The fact 


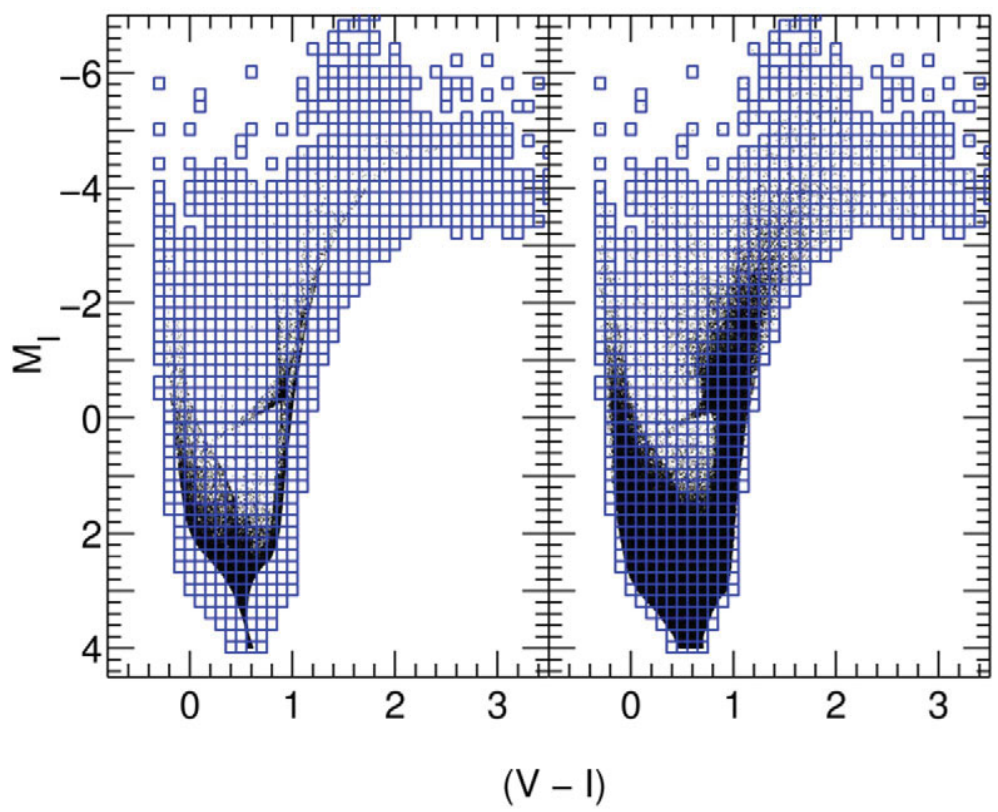

Figure 3. Set of boxes "grid" for the fCMD (left panel) and sCMD (right panel). It is shown a number of 900 boxes. A size of $\Delta(V-I)=0.1$ and $\Delta(I)=0.2$ have been defined for each box.

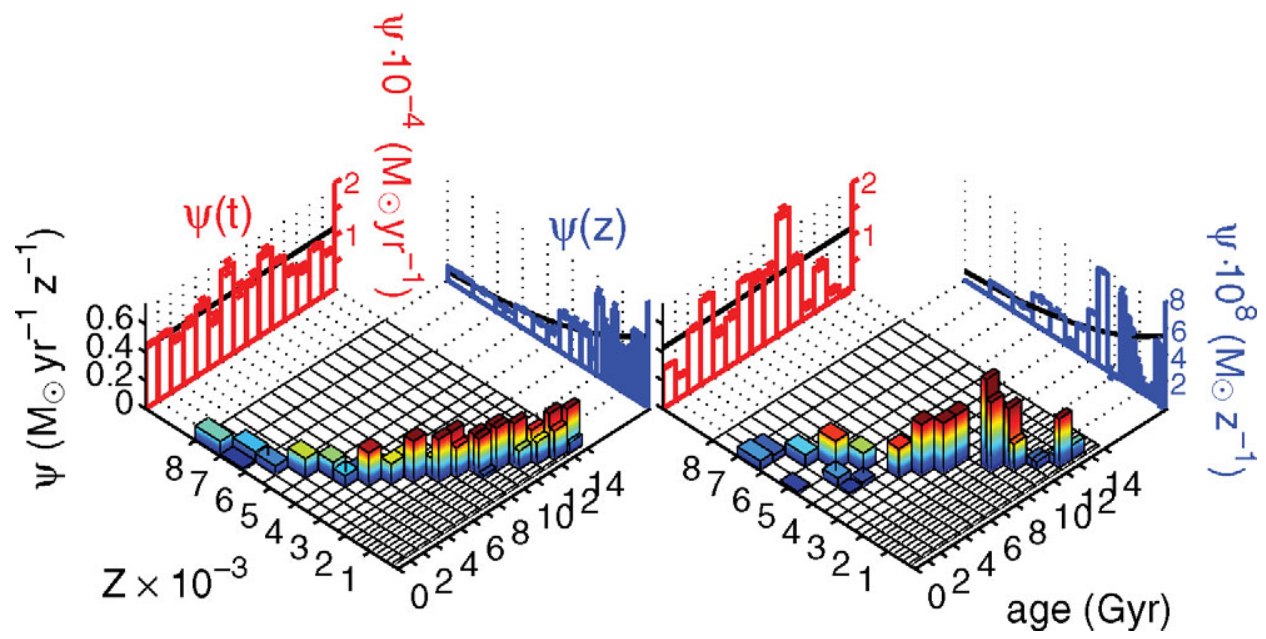

Figure 4. Solutions of $\psi(t, z)$ for the "grid" parameterization (see Fig. 3). Left panel: solution obtained using the same stellar evolution library (Padua) for the fake and the mother synthetic populations. Right panel: solution obtained using different stellar evolution libraries for the fake (Padua) and the mother synthetic (BaSTI-Teramo) populations. Figure labels and colors are as in Fig. 3.

that both are very similar indicates that IAC-pop has a good degree of self-consistency and robustness. The second case, in which different libraries are used, is a more realistic one. It also simulates the departure to be expected from the real SFH of a system due to uncertainties in current generations of stellar evolution models. 


\section{Final remarks and conclusions}

Summarizing, IAC-pop is a program designed to solve the SFH of a complex stellar population system, like a galaxy, from the analysis of the CMD. To this purpose, IAC-pop uses a genetic algorithm (Charbonneau 1995) to minimize a Mighell $\chi^{2}$ merit function (Mighell 1999) comparing the star distribution in observed and model CMDs. The code main characteristics can be sketched as follows:

- The code needs the computation of only a single global (mother) synthetic CMD. As many simple population model CMD as necessary are later extracted from it.

- It is designed to solve simultaneously for age and metallicity distributions; i.e. for the star formation rate and the chemical enrichment law.

- The code uses a parameterization of the observed and model CMDs. This is done by dividing the CMDs in several boxes by a grid and counting out the stars in each one. This is, in fact, the information provided to the code.

- The former implies that the code is not restricted to solve the problem of the SFH, but it is of general application to problems in which a similar parameterization can be applied.

- It is important to note that observational effects (crowding, blending, completeness, etc) must be simulated in the synthetic data prior to run IAC-pop. Natural places are in the original synthetic CMD or in the final parameterization.

- The final solution is provided as a linear combination of positive or null coefficients of the input simple population models. A genetic algorithm is used at this point to minimize the aforementioned Mighell $\chi_{\gamma}^{2}$.

- In its current version, IAC-pop provides the best solution $\chi_{b e s t}^{2}$ for one or several runs. It also provides two error estimates: the first one as the $\sigma$ values of solutions placed at $\chi_{\text {best }}^{2}+1$, and the second one as the $\sigma$ values of the solution obtained after random poissonian deviation of the input parameterization.

The program is offered for free use and can be retrieved at the URL site: iac-star.iac.es/iac-pop, with the only requirement of referencing Aparicio \& Hidalgo (2007) paper and acknowledging the IAC in any derived publication.

\section{Acknowledgements}

Financial support has be provided by the IAC Research Division. The authors are funded by the IAC (grant P3/94) and by the Science and Technology Ministry of the Kingdom of Spain (grant AYA2004-06343).

\section{References}

Aparicio, A., \& Gallart, C. 1995, AJ, 110, 2105

Aparicio, A., \& Gallart, C. 2004, AJ, 128, 1465

Aparicio, A., \& Hidalgo, S.L. 2007, $A J$, submitted

Aparicio, A., Gallart, C., \& Bertelli, G. 1997, AJ, 114, 680

Bertelli, G., Bressan, A., Chiosi, C., Fagotto, F., \& Nasi, E. 1994, A\&AS, 106, 275

Castelli, F., \& Kurucz, R.L. 2002, in "Modeling of Stellar Astmospheres", IAU Symp. 210 (N.E.

Piskunov et al. (eds), in press

Charbonneau, P. 1995, ApJS, 101, 309

Dolphin, A. E. 1997, New Astron., 2, 397

Kroupa, P., Tout, C.A., \& Gilmore, G. 1993, MNRAS, 262, 545

Pietrinferni, A., Cassisi, S., Salaris, M., \& Castelli, F. 2004, ApJ, 612, 168 


\section{Discussion}

VAZDEKIS: Is Phoenix a prototype case for the SFH that you obtain?.

ApARICIO: No. What is found in LG dwarf galaxies is rather that each one has its own particular SFH.

Hyun-Chul LeE: Is your binary consideration a simple mixture of single stars or do you consider all the mass transfer and interactions between binaries?.

ApARICIO: Yes, it is a simple mix of single stars with the same age and metallicity.

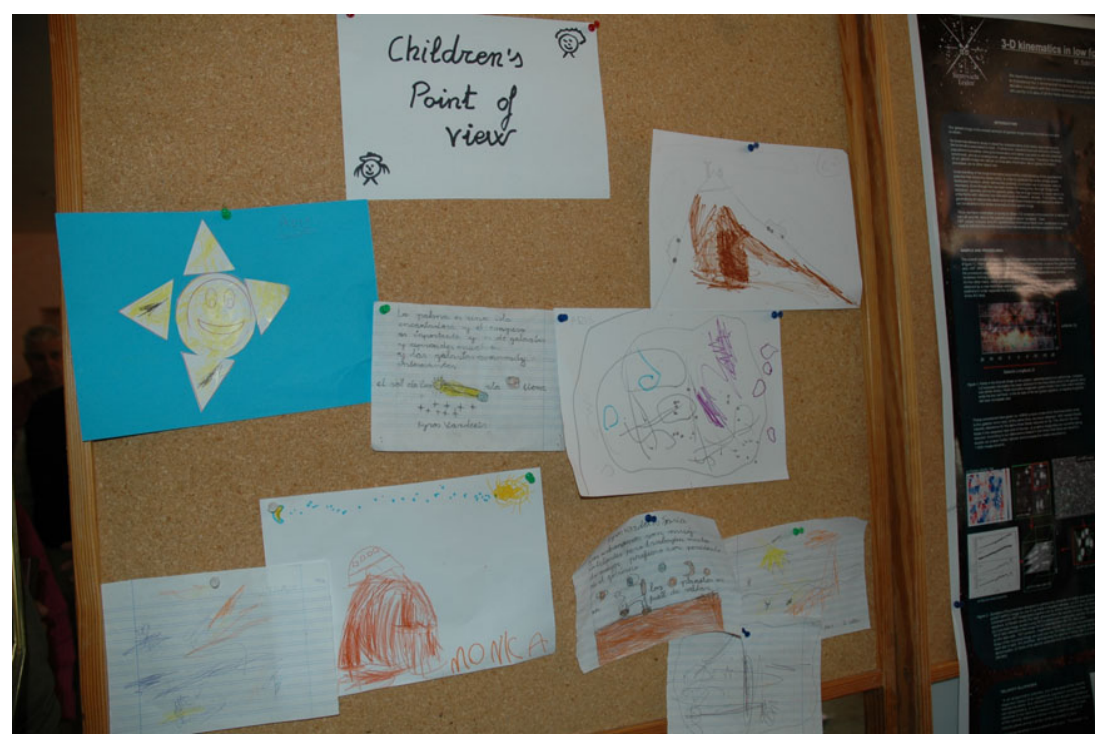

The children's point of view.

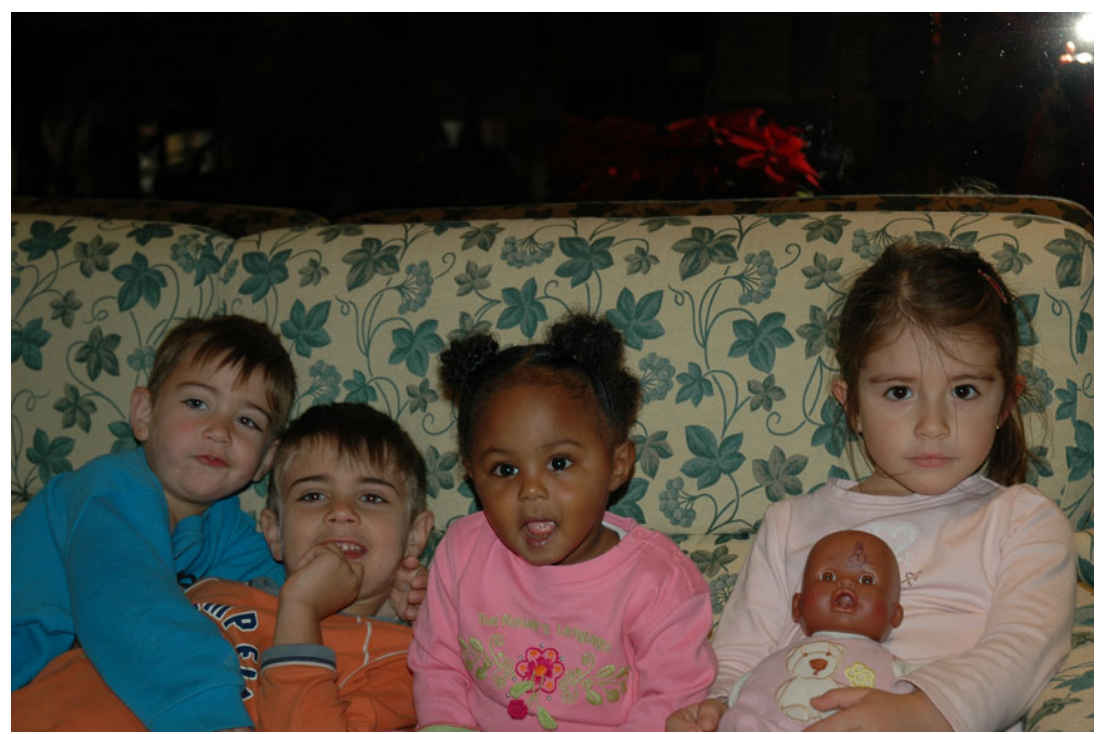

